\title{
Case Report: Madariaga Virus Infection Associated with a Case of Acute Disseminated Encephalomyelitis
}

\author{
Kathia Luciani, Iván Abadía, Alex O. Martínez-Torres, Julio Cisneros, Ilka Guerra, Mariana García, \\ Dora Estripeaut, and Jean-Paul Carrera* \\ Division of Pediatric Infection Disease and Division of Pediatric Neurology, Hospital del Niño, Panama City, Panama; Department of Research in \\ Virology and Biotechnology, Gorgas Memorial Institute of Health Studies, Panama City, Panama; School of Medicine, Columbus University, \\ Panama City, Panama; Department of Microbiology and Parasitology, University of Panama, Panama City, Panama
}

\begin{abstract}
We present the first report of a pediatric case of acute disseminated encephalomyelitis (ADEM) associated with Madariaga virus infection (MADV, Alphavirus, Togaviridae; formerly known as South American variants of eastern equine encephalitis virus [EEEV]) in a patient of the 2010 alphaviral epidemic reported in Panama. The patient was admitted to the Hospital del Niño in Panama City with suspected meningitis, exhibited with decreased alertness and disorientation in space and time, hemiparesis, and left Babinski sign. The patient was transferred to the intensive care unit and treated with aciclovir and methylprednisolone. The magnetic resonance imaging (MRI) of the brain revealed multiple hyperintense lesions at $\mathrm{T}_{2}$-weighted images (T2) and fluid-attenuated inversion recovery (FLAIR) on the cortical-subcortical level. Sera samples obtained on days 6 and 12 were immunoglobulin M (IgM) positive for MADV. The findings on the clinical and cerebrospinal analyses, rapid symptom progression as well as neuroimaging, and serologic studies support our diagnosis. Our results suggest that MADV should be included in the etiologic differential diagnosis of ADEM in endemic countries.
\end{abstract}

\section{INTRODUCTION}

To our knowledge, until the 2010 human and equine encephalitis outbreak in Panama, Madariaga virus had not been associated with severe or neurological human disease in Latin America. ${ }^{1,2}$ Acute disseminated encephalomyelitis (ADEM) is an autoimmune process frequently associated with exanthematic viruses, bacterial etiology, or immunizations. ADEM is characterized by acute encephalopathy, associated with multifocal neurological symptoms, as a consequence of the demyelination of the white matter and spinal cord, posterior to an antigen challenge. ${ }^{3}$ It usually starts with fever, general discomfort, headache, nausea, and vomiting and progresses rapidly to encephalopathy, associated with symptoms such as ataxia (18$65 \%)$, hemiplegia $(76 \%)$, cranial nerve paralysis $(22-45 \%)$, optic neuritis (7-23\%), language alterations (5-21\%), and hemihypesthesia $(2-3 \%))^{3}$ Convulsions are infrequent in adults, and more frequent in those $<5$ years of age. Between $11 \%$ and $16 \%$ of the patients may present secondary respiratory insufficiency. ${ }^{4}$ The presentation is usually monophasic but several studies describe relapses in $5.5-2.1 \%$ of cases. 5,6 Radiological and clinical criteria are necessary to diagnose. ${ }^{4,7}$ The etiological diagnosis continues to be a challenge, in most cases the causal agent is never established. The requested studies must be prioritized according to season, geographical area, exposure history, and clinical history. ${ }^{4}$

Here, we present a description of a pediatric patient from the 2010 encephalitis outbreak who presented with ADEM associated with an MADV infection.

\footnotetext{
* Address correspondence to Jean-Paul Carrera, Department of Research in Virology and Biotechnology, Gorgas Memorial Institute of Health Studies, School of Medicine Columbus University, Justo Arosemena Avenue, 39th Street, Panama City, Panama 0816-02593. E-mail: jpcarrera@gorgas.gob.pa
}

\section{CASE REPORT}

The case presented here involves a 9-year-old schoolboy from the eastern Panamanian province of Darién who was admitted to the Hospital del Niño in Panama City with suspected meningitis. He presented with a history of 2 days of unquantified fever, headache, and vomiting associated with three widespread tonic-clonic seizure episodes and a history of upper respiratory tract infection 7 days before the onset of symptoms.

Physical examination revealed heart rate: 120 cycles/min $(\mathrm{cpm})$, respiratory rate: $20 \mathrm{cpm}$, temperature: $39^{\circ} \mathrm{C}$, alert, and stiff neck without other symptoms of meningeal irritation. Hematology results showed leukocytosis 19,500; neutrophils $93 \%$; bands $3 \%$; lymphocytes $1 \%$ and monocytes $3 \%$; platelets 289,000; and C-reactive protein $18.1 \mathrm{mg} / \mathrm{dL}$. Cerebrospinal fluid (CSF) appeared clear, colorless and contained 146 leukocytes/ $\mathrm{mm}^{3}$ with mononuclear predominance $97 \%, 6$ red blood cells/ $\mathrm{mm}^{3}$, total proteins $274 \mathrm{mg} / \mathrm{dL}$, and glucose $69 \mathrm{mg} / \mathrm{dL}$.

During the next 12 hours, the patient exhibited decreased alertness and disorientation in space and time, hemiparesis, and left Babinski sign and was transferred to the intensive care unit; treatment with aciclovir and methylprednisolone was started. The differential diagnoses considered were encephalitis versus encephalomyelitis. During his hospitalization, 10 other children from the same geographical region were admitted, with clinical signs of encephalitis, six of them were confirmed as MADV infections, three had venezuelan equine encephalitis virus (VEEV) infection, and one was dual infected with both viruses; all these cases were confirmed by immunoglobulin M (IgM) serology. ${ }^{1}$ The National Veterinary Epidemiological Surveillance System reported equine deaths in the region, and Gorgas Memorial Institute or the University of Texas Medical Branch confirmed equine MADV infections. ${ }^{1}$

Magnetic resonance imaging (MRI) of the brain revealed multiple hyperintense lesions at $\mathrm{T}_{2}$-weighted images (T2) and fluid-attenuated inversion recovery (FLAIR) on the corticalsubcortical level, more evidently at the frontal and parietal areas of the right hemisphere as well as bilateral compromise 

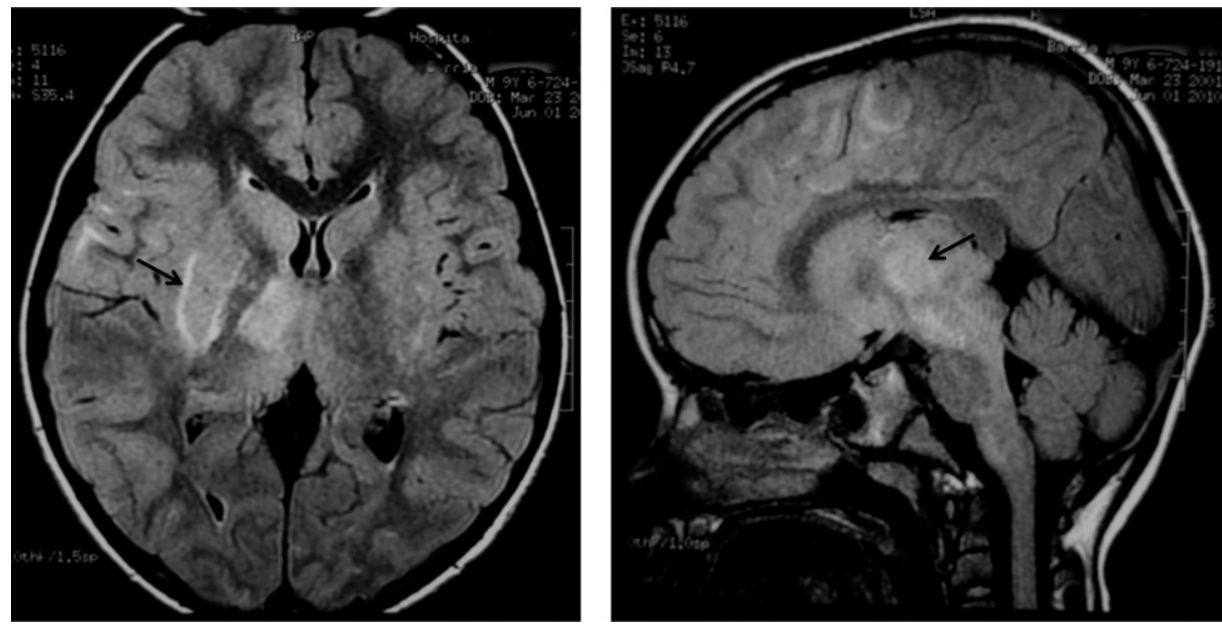

FIGURE 1. Axial and sagittal $\mathrm{T}_{2}$-weighted images (T2) and fluid-attenuated inversion recovery (FLAIR) magnetic resonance shows multiple hyperintense lesions at the cortical-subcortical level, most evident in the frontal and parietal right hemisphere. These lesions also affected the basal ganglia bilaterally, the right mesencephalic, and thalamic region.

of the basal nuclei and the right thalamic mesencephalic region. (Figure 1). Serum samples were tested for antibodies against MADV and VEEV using a capture IgM enzymelinked immunosorbent assay (ELISA); later confirmed with plaque reduction neutralization test (PRNT) as described previously in a study. ${ }^{5}$ Sera samples obtained on days 6 and 12 post-presentation were only positive for $\operatorname{IgM}$ antibodies to MADV, and IgM antibodies were not detected on day 44. A PRNT performed in the sample obtained on day 6 was MADV positive; no samples from days 12 and 44 were available for PRNT confirmation. The virus was not isolated.

The progression of recovery was satisfactory; there was rapid neurological recovery without mechanical support. After 4 days of steroid treatment, the patient was alert and oriented, but still had left hemiparesis. He was discharged 10 days after admission with an improvement in the condition of hemiparesis. At the follow-up appointment a month after his discharge, he was asymptomatic without evidence of long-term neurological effects and indications of a progressive decrease of steroids.

\section{DISCUSSION}

Eight decades after the first isolation of MADV in Latin America from horses, the first documented human outbreak was reported in Panama in 2010. ${ }^{1}$ ADEM is generally a monophasic demyelinating disease of the central nervous system, which occurs mainly during the pediatric age and frequently happens after an infection or vaccination. ${ }^{3,6}$ However, species of the eastern equine encephalitis virus (EEEV) antigenic complex have never been associated as ADEM cause, even in North America where human neurologic disease is more common. ${ }^{8}$

Here, we report a pediatric case of ADEM in Panama, associated with a confirmed case of MADV infection. The patient presented a prodromal phase accompanied by fever, headache, and vomiting. ADEM begins with fever, general discomfort, headache, nausea, and vomiting and progresses rapidly to encephalopathy, associated with ataxia, hemiplegic, cranial nerve paralysis, optical neuritis, language alterations, and hemihypesthesia. ${ }^{3,9}$ Seizures are frequent in patients $<5$ years of age. Diagnosis is based on radiological and clinical criteria. ${ }^{3,10}$ In our patient, we observed white cell count and protein elevation in CSF. In patients with ADEM, an elevation of white blood cells in CSF is regularly found and peripheral leukocytes, platelets, and the erythrocyte sedimentation rate are usually elevated.

The patient's axial and sangittal MRI shows a signal increase in FLAIR on multiple lesions in the thalamic-mesencephalic region and the right subcortical frontal-parietal region. The MRI usually shows patch lesions with poorly defined margins of signal increase in T2 and FLAIR. The patient's MRI was consistent with ADEM, showing multiple lesions that mainly affected the white matter, found in both hemispheres and in the mesencephalic region, described as hypertense in the T2 and FLAIR sequences and hypotense at T1, which supports its inflammatory nature. ${ }^{6}$

Because ADEM is an acute inflammatory disease, etiological agents are not typically present by the time neurologic disease appears, and etiologic diagnosis continues to be a challenge. A good clinical history is essential to establish the diagnosis. The overlapping circulation of MADV and VEEV in Panama underscores another diagnostic challenge in Latin American endemic countries. Cross IgM or IgG reaction due to heterologous antibodies can be observed; in this case, PRNT is an invaluable tool for laboratory confirmation. ${ }^{1}$ In this patient, we observed seroconversion from positive to negative IgM and neutralizing antibodies against only MADV.

Although we could not isolate the MADV from this patient, phylogenetic studies performed on two isolated strains during the 2010 outbreak indicated that the causative agent was a Panamanian enzootic strain of MADV. ${ }^{1}$

Unlike other MAD human cases of the 2010 outbreak, the case we presented here was the only one with ADEM clinical presentation. ${ }^{1}$ This fact may support the hypothesis of genetic predisposition to develop ADEM; however, genome-wide association studies are required from a large populationbased research. $^{11}$

There is no standard treatment for ADEM. Steroids are used in high doses and diverse protocols use methylprednisolone or dexamethasone for 3-5 days, followed by oral steroids 
for 4-6 weeks. ${ }^{3,9}$ Immune globulin intravenous injection (IGIV) has been used successfully but there are no comparative or clinical randomized studies. Its use on relapse patients or patients with no improvement after a course of steroids has been described. ${ }^{12,13}$ In this patient, the resolution of the infection/condition was complete with the use of steroids. Studies on children with ADEM show that two-thirds recover completely over the course of several weeks. ${ }^{8,11}$

In 2007, the International Pediatric Multiple Sclerosis Study Group proposed the presence of encephalopathy, multifocal affectation, and the absence of previous demyelinating events as diagnostic criteria for ADEM, all of which are present in this patient. ${ }^{3,10}$ The diagnosis of ADEM in this patient was supported by remission of signs following treatment with intravenous methylprednisolone. To our knowledge, the case we presented here is the first case of ADEM recorded in the medical literature after MADV infection.

Received December 31, 2014. Accepted for publication March 4, 2015. Published online April 13, 2015.

Acknowledgments: We thank Scott Weaver and Robert B. Tesh for scientific and diagnosis advice during the epidemic, Rodrigo Villalobos for suggestions on the manuscript, and Amanda Gabster for English revision of the manuscript.

Financial support: This study received financial support from the surveillance program of the Department of Research in Virology and Biotechnology by contract from the Secretaría Nacional de Ciencia, Tecnología e Innovación, Panama (60-4-FID09-103, to J-PC). JeanPaul Carrera is also paid by research contract CU-VIP-P-003-2013 from Columbus University.

Authors' addresses: Kathia Luciani and Dora Estripeaut, Division of Pediatric Infection, Hospital del Nino, Panama City, Panama, E-mails: kathialuciani@gmail.com and destripeaut@gmail.com. Iván Abadía, Division of Pediatric Neurology, Hospital del Nino, Panama City, Panama, E-mail: iabadia@hotmail.com. Laboratorio de Microbiología Experimental y Aplicada - LAMEXA, Vicerrectoría de Investigación Postgrado, Universidad de Panamá, E-mail: amartinet13@hotmail. com. Julio Cisneros, Ilka Guerra, and Mariana García, Department of Virology and Biotechnology Research, University of Panama, Panama City, Panama, E-mails: jygcisneros@cwpanama.net, ilkaguerra@yahoo. com, and garciameijide@gmail.com. Jean-Paul Carrera, Department of Research in Virology and Biotechnology, Panama City, Panama, and School of Medicine Columbus University, Panama City, Panama, E-mail: jean1450@gmail.com.

\section{REFERENCES}

1. Carrera JP, Forrester N, Wang E, Vittor AY, Haddow AD, Lopez-Verges S, Abadía I, Castaño E, Sosa N, Báez C, Estripeaut D, Díaz Y, Beltrán D, Cisneros J, Cedeño HG, Travassos da Rosa AP, Hernandez H, Martínez-Torres AO, Tesh RB, Weaver SC, 2013. Eastern equine encephalitis in Latin America. $N$ Engl J Med 369: 732-744.

2. Arrigo NC, Adams AP, Weaver SC, 2010. Evolutionary patterns of eastern equine encephalitis virus in North versus South America suggest ecological differences and taxonomic revision. J Virol 84: 1014-1025.

3. Tenembaum S, Chitnis T, Ness J, Hahn JS, the International Pediatric MS Study Group, 2007. Acute disseminated encephalomyelitis. Neurology 68 (Suppl 2): S23-S36.

4. Tenembaum S, Chamoles N, Fejerman N, 2002. Acute disseminated encephalomyelitis: a long-term follow-up study of 84 pediatric patients. Neurology 59: 1224-1231.

5. Beaty BJ, Calisher CH, Shope RE, 1995. Diagnostic procedures for viral, rickettsial and chlamydial infections. Lennette $\mathrm{EH}$, Lennette DA, Lennette ET, eds. Arboviruses. Washington, DC: American Public Health Association, 189-212.

6. Menge T, Hemmer B, Nessler S, Wiendl H, Neuhaus O, Hartung HP, Kieseier BC, Stüve O, 2005. Acute disseminated encephalomyelitis: an update. Arch Neurol 62: 1673-1680.

7. Murthy SN, Faden HS, Cohen ME, Bakshi R, 2002. Acute disseminated encephalomyelitis in children. Pediatrics 110: e21.

8. Deresiewicz RL, Thaler SJ, Hsu L, Zamani AA, 1997. Clinical and neuroradiograph manifestations of eastern equine encephalitis. N Engl J Med 336: 1867-1874.

9. Erazo-Torricelli R, 2006. Acute disseminated encephalomyelitis in children. Rev Neurol 42 (Suppl 3): S75-S82.

10. Krupp LB, Banwell B, Tenembaum S, the International Pediatric MS Study Group, 2007. Consensus definitions proposed for pediatric multiple sclerosis and related disorders. Neurology 68 (Suppl 2): S7-S12.

11. Khor CC, Bich CTN, Pang J, Davila S, Long HT, Ong RT, Dunstan SJ, Wills B, Farrar J, Van Tram T, Gan TT, Binh NT, Tri le T, Lien le B, Tuan NM, Tham NT, Lanh MN, Nguyet NM, Hieu NT, Van N Vinh Chau N, Thuy TT, Tan DE, Sakuntabhai A, Teo YY, Hibberd ML, Simmons CP, 2011. Genome-wide association study identifies susceptibility loci for dengue shock syndrome at MICB and PLCE1. Nat Genet 43: 1139-1141.

12. Imataka $S$, Arisaka $O, 2014$. An infant with steroid refractory cytomegalovirus-associated ADEM who responded to inmunoglobulin therapy. Eur Review Med Pharmacol Sci 18: 2148-2151.

13. Erol I, Ozkale Y, Alkan O, Alehan F, 2013. Acute disseminated encephalomyelitis in children and adolescents: a single center experience. Pediatr Neurol 49: 266-273. 